\title{
Prognostic value of cancer stem cells, epithelial-mesenchymal transition and circulating tumor cells in lung cancer
}

\author{
G. PIROZZI ${ }^{1}$, V. TIRINO ${ }^{2}$, R. CAMERLINGO ${ }^{1}$, A. LA ROCCA ${ }^{3}$, N. MARTUCCI ${ }^{3}$, \\ G. SCOGNAMIGLIO ${ }^{4}$, R. FRANCO ${ }^{4}$, M. CANTILE ${ }^{4}$, N. NORMANNO ${ }^{1}$ and G. ROCCO ${ }^{3}$ \\ ${ }^{1}$ Department of Experimental Oncology, National Cancer Institute, Pascale Foundation, I-80131 Naples; \\ ${ }^{2}$ Department of Experimental Medicine, Second University of Naples, I-80138 Naples; ${ }^{3}$ Department of \\ Thoracic Surgery and Oncology, National Cancer Institute, Pascale Foundation, I-80131 Naples; \\ ${ }^{4}$ Department of Pathology, National Cancer Institute, Pascale Foundation, I-80131 Naples, Italy
}

Received August 28, 2012; Accepted October 23, 2012

DOI: 10.3892/or.2013.2294

\begin{abstract}
The epithelial-mesenchymal transition (EMT) is a program involved in embryonic development that is often activated during cancer invasion and metastasis. CD133 is the main marker identifying cancer stem cells (CSCs) in lung cancer. Circulating tumor cells (CTCs) are demonstrated to be useful as a biomarker for the diagnosis and treatment of cancer. The aim of this study was to correlate EMT, CSCs and CTCs with patient prognosis to verify whether they can contribute to better stratification of lung cancer patients at risk for recurrent and metastatic disease. Pulmonary venous blood was drawn after major pulmonary surgery in 45 patients with resectable non-small cell lung cancer (NSCLC) in order to identify CTCs. For the same patients, we also constructed prognostic lung tissue microarrays (TMA) for CD133 and c-kit and evaluated CSC and EMT markers using flow cytometry. Cytokeratin-positive cells were detectable in $11(23.9 \%)$ cases. c-kit expression was heterogeneous in prognostic TMAs while CD133 expression was low or absent which was also confirmed by flow cytometry and RT-PCR. Flow cytometric analysis showed that the mean percentage of cells with CD133 expression was 1.6\%. CD90 and CD326 markers were co-expressed with a mean percentage of $10.41 \%$. When CD133 and CD90/CD326 expression was correlated with follow-up, CD133 showed a higher correlation with deceased patients when compared with CD90/ CD326 co-expression (32.5 vs. 9.5\%). CD133 expression demonstrated a strong significant association with patients exhibiting progressive disease when compared to CD90/ CD326 expression (15 vs. $7.1 \%$ ). CD133 may be significantly
\end{abstract}

Correspondence to: Dr Giuseppe Pirozzi, Department of Experimental Oncology, National Cancer Institute, Pascale Foundation, Via Mariano Semmola, I-80131 Naples, Italy

E-mail: pinopiro@tin.it

Key words: epithelial-mesenchymal transition, cancer stem cells, circulating tumor cells, prognosis associated with invasion and metastatic spread of NSCLC. The co-expression of CD90, CD326 and CD133 has definite prognostic value in patients with NSCLC.

\section{Introduction}

Lung cancer is the most common cause of cancer-related death in the world at present (1). Currently, the survival of lung cancer patients is poor with only $15 \%$ of patients surviving 5 years follosing diagnosis. Although new chemotherapy agents and radiotherapy have improved the survival and quality of life of patients, the overall effect in the last decade has been mainly on palliation rather than reduction in mortality. Two important hypotheses have been postulated to explain the genesis, formation, growth and metastasis of epithelial cancer: the role of cancer stem cells (CSCs) or tumor-initiating cells (TICs) and the involvement of 'so called' epithelial-mesenchymal transition (EMT). The CSC hypothesis asserts that only a small subset of cells within a tumor has the ability of both tumor initiation and sustaining tumor growth $(2,3)$. These cells, that express stemness markers, are capable of forming floating spheres in serum-free medium, a property associated with stem cells and are able to differentiate into an aberrant cell phenotype constituting tumor heterogeneity (4). There are two basic concepts that support the hypothesis that CSCs originate from normal tissue stem cells. First of all, CSCs have normal stem cell features such as self-renewal, differentiation, drug resistance and migration capacity. Moreover, the longevity of stem cells makes them susceptible to accumulating genetic and epigenetic damages so as to make them a suitable candidate for the emergence of neoplastic transformation. CSCs are the only cells that are capable of generating tumors similar to the original patient specimens when transplanted into immunocompromised mice such as NOD/SCID mice. Finally, CSCs have been identified in a variety of solid tumors including glioblastomas (5), breast (6) and lung cancer $(7,8)$.

During tumorigenesis, subsets of tumor cells localized within the primary tumor may acquire features of invasiveness and motility and enter blood or lymph vessels. Mechanisms 
involved in this process are still under investigation; however, they have been reported to be linked to variable interactions between tumor cells and their surrounding stroma, including response to hypoxia and metalloproteinase-dependent invasion into surrounding tissue, (neo-)vascularization of the tumor (9), as well as gain of a phenotype revealing signatures of EMT observed in at least a subpopulation of tumor cells with certain 'stemness' properties (10). EMT is a key program in embryonic development, often activated during cancer invasion and metastasis and by which cells undergo a morphological switch from the epithelial polarized phenotype to the mesenchymal fibroblastoid phenotype. As a result of EMT, epithelial cells lose their defined cell-cell/cellsubstratum contacts and their structural/functional polarity, and they become spindle-shaped and morphologically similar to activated fibroblasts (11) by downregulation of epithelial differentiation markers including cytokeratins and E-cadherin and transcriptional induction of mesenchymal markers such as vimentin, fibronectin and $\mathrm{N}$-cadherin. CTCs can infiltrate and survive in and colonize to distant organs (12). Recent advances in this field are supportive for the early dissemination model of metastasis, through the observation that disseminated tumor cells (DTCs) isolated from bone marrow or lymph nodes display disparate changes on all levels of genomic resolution as compared to primary tumor cells (13). Cancer cell dissemination may be followed by a dormancy period before relapse in one or more organs (14). Current research on DTCs and CTCs presents a challenge, as these cells are well-defined targets for understanding tumor biology and tumor cell dissemination in cancer patients (15), and may open new avenues for the early detection of metastatic spread and its successful treatment.

The aim of the present study was to correlate EMT markers, CTCs and CSCs with clinicopathological parameters and follow-up of patients with NSCLC to verify whether these markers contribute to accurate stratification of patients at risk for recurrent and metastatic disease.

\section{Materials and methods}

Patients and follow-up. Forty-five consecutive patients undergoing resection for primary NSCLC were enrolled. As previously described (16), in all cases, preoperative staging was performed in order to exclude patients with advanced disease. Generally, lung resection consisted of lobectomy or pneumonectomy with systematic mediastinal lymphadenectomy. Immediately after surgical resection, tumor-draining pulmonary vein stumps were punctured to obtain blood samples. Follow-up studies included physical examination, chest X-ray, and blood tests at a 3-month interval and an additional thoracic chest and abdomen (CT) scan, along with flexible bronchoscopy at a 6-month interval. Thus, relapses and deaths were recorded in our files. Ethics committee approval was provided in all instances (IRB approval no. 556).

Demographics. Patient characteristics are documented in Table I. There were 14 female patients. The median age at the time of surgery was 74.2 years (range, 48-81). The median follow-up duration was 16.3 months (range, 1-26). Pathological
Table I. Characteristics of patients enrolled in this study and distribution of CTCs and CD133.

\begin{tabular}{|c|c|c|}
\hline & $\begin{array}{c}\text { Patients }(\%) \\
\quad(\mathrm{n}=45)\end{array}$ & $\begin{array}{c}\text { Relation to CD133 } \\
\text { positivity } \\
\text { P-value }\end{array}$ \\
\hline \multicolumn{3}{|l|}{ Age (years) } \\
\hline$<60$ & 26.7 & 0.656279 \\
\hline$\geq 60$ & 73.3 & \\
\hline \multicolumn{3}{|l|}{ Gender } \\
\hline Male & 68.9 & 1 \\
\hline Female & 31.1 & \\
\hline \multicolumn{3}{|l|}{ Stage } \\
\hline I & 71.1 & 0.56015 \\
\hline II & 17.8 & \\
\hline III & 11.1 & \\
\hline \multicolumn{3}{|l|}{ Histological type } \\
\hline Squamous cell & 55.6 & 0.543478 \\
\hline Adenocarcinoma & 33.3 & \\
\hline Large-cell & 11.1 & \\
\hline \multicolumn{3}{|l|}{ Relapse } \\
\hline No & 68.9 & 0.136 \\
\hline Yes & 31.1 & \\
\hline \multicolumn{3}{|l|}{ Status } \\
\hline Alive & 71.1 & 0.26018 \\
\hline Alive with disease & 15.6 & \\
\hline Death due to tumor & 13.3 & \\
\hline \multicolumn{3}{|l|}{ Circulating cells } \\
\hline Absence & 75.6 & 0.588 \\
\hline Presence & 24.4 & \\
\hline \multicolumn{3}{|l|}{ CD117 } \\
\hline Absence & 73.3 & 0.044 \\
\hline Presence & 26.7 & \\
\hline
\end{tabular}

stage I was recorded in 32 patients, stage II in 8 patients and stage III in 5 patients. Relapses were recorded in 11 cases, with a mean time of 14 months. At the end of the follow-up, death was observed in 6 cases, 32 patients were alive without disease and 7 patients were alive with disease.

Histology of primary tumors. All cases were reviewed according to WHO classification. In particular, pathological stage, histological type and when applicable cancer grade were determined.

Isolation of cancer cells from NSCLC biopsies and flow cytometry. Fresh NSCLC biopsies were minced with scissors, filtered through 40-mm nylon meshes and subsequently analyzed for EMT and CSC markers using flow cytometry. At least 200,000 cells were incubated with $1 \mu \mathrm{g} / \mu \mathrm{l}$ of fluorescent-labelled monoclonal antibodies or respective isotype controls at $4^{\circ} \mathrm{C}$ for $30 \mathrm{~min}$ in the dark. After incubation, the 
samples were washed and analyzed by flow cytometry using a FACSAria II (Becton-Dickinson, USA). The antibodies used were: CD133/2 PE (Miltenyi Biotec S.r.l., Calderara di Reno, Bologna, Italy), CSC marker CD326 PE (EpCAM, Miltenyi Biotec), CD90 FITC (BD Pharmingen, Buccinasco, Milan, Italy), mesenchymal marker CD45 CY (BD Pharmingen) and leukocyte marker. All data were analyzed using DIVA 6.6 software.

Isolation of mononuclear cells from pulmonary venous blood and CTC identification. The tumor-draining pulmonary vein was punctured subsequent to thoracotomy and prior to intrathoracal preparation for lung resection. Pulmonary venous blood $(10 \mathrm{ml})$ was drawn into a sterile syringe. The obtained pulmonary venous blood, yielding between $5 \times 10^{6}$ and $6 \times 10^{7}$ (mean, $2.5 \times 10^{7}$ ) mononuclear cells, was centrifuged through $20 \mathrm{ml}$ Ficoll-Hypaque for $30 \mathrm{~min}$ at $1,200 \mathrm{rpm}$. The interface layer, which contains mononuclear cells, was collected and brought to a final concentration of $10^{6}$ cells $/ \mathrm{ml}$. The obtained cells were fixed with Cytolit ${ }^{\mathrm{TM}}$ solution and then processed according to the ThinPrep2000 ${ }^{\mathrm{TM}}$ method (Cytyc Corp., Marlborough, MA).

Occult tumor cells of pulmonary venous blood were detected by immunohistochemical staining using pan-cytokeratins. Antigen retrieval was performed on all slides obtained by the ThinPrep2000 method. The first step of antigen retrieval lasted for $30 \mathrm{~min}$ at $\sim 100^{\circ} \mathrm{C}$ with a citrate-based buffer (Leica Bond Epitope retrieval solution, $\mathrm{pH}$ 5.9-6.1). CK 8/18/19 (pan-CK) mouse monoclonal antibody (clone A45-B/B3, 1:100 dilution; Miltenyi Biotec $\mathrm{GmbH}$, Gladbach, Germany) was applied and incubation was carried out for $60 \mathrm{~min}$, followed by a washing step to remove any excess antibody. The antibody was visualized using the peroxidase detection system (RE7120-K; Novocastra, Newcastle, UK) and diaminobenzidine (DAB) as contrasting chromogen.

TMA construction. As previously described, a tissue microarray (TMA) was constructed using the most representative areas from each single case (16) using a semiautomated tissue arrayer (Galileo TMA CK 3500).

Immunohistochemistry. Primary antibodies included antihuman CD133 (AC133, dilution 1.150; Miltenyi Biotec), c-kit (polyclonal, diluition 1:500; Dako) and CD90 (EPR3132, dilution 1:150; Lifespan). Antigen retrieval was performed by microwave pretreatment in $0.01 \mathrm{M}$ citrate buffer for $10 \mathrm{~min}$. Sections were incubated with mouse anti-rabbit or goat anti-mouse secondary immunoglobulin ( $\mathrm{IgG}$ ) biotiny-lated secondary antibody for $30 \mathrm{~min}$. Immunoreactivity was visualized by means of avidin-biotin-peroxidase complex kit reagents (Novocastra) as the chromogenic substrate. Finally, sections were weakly counterstained with hematoxylin and mounted. Appropriate inner cells were considered as controls. For each antibody, cytoplasmic and membranous staining was recorded. Tissues were scored semi-quantitatively by evaluating the proportion of positive tumor cells over the total number of tumor cells (percentage of positive tumor cells per tissue microarray punch). Negative, low expression and high expression cases were recorded when neoplastic cells expressing chemokine receptor were between 0 and $10 \%,<30 \%$ and $>30 \%$, respectively.
$C D 133 q R T-P C R$. Total RNA was isolated from frozen biopsies from our Institutional Bio-Bank, using RNeasy Mini kit (Qiagen GmbH, Hilden, Germany) following the manufacturer's instructions. Samples were treated with RNase-free DNase (Qiagen $\mathrm{GmbH}$ ) to prevent amplification of genomic DNA. A total of $1 \mu \mathrm{g}$ RNA was subjected to cDNA synthesis for $1 \mathrm{~h}$ at $37^{\circ} \mathrm{C}$ using the Ready-To-Go You-Primer First-Strand Beads kit (code 27-9264-01; Amersham Biosciences Europe GmbH, Freiburg, Germany) in a reaction mixture containing $0.5 \mu \mathrm{g}$ random hexamers (GeneAmp RNA PCR random hexamers set N808-0127; Applied Biosystems, Foster City, CA).

Quantitative RT-PCR was performed using a LightCycler system (Roche Molecular Biochemicals, Mannheim, Germany) using TaqMan ${ }^{\circledR}$ analysis. In this system, all reactions were run in glass capillaries with LightCycler TaqMan Master Mix (code 04735536001; Roche Molecular Biochemicals), $10 \mu \mathrm{l}$ in a volume of $20 \mu \mathrm{l}$ containing $2 \mu \mathrm{l}$ of cDNA and $1 \mu \mathrm{l}$ of specific TaqMan Gene Expression Assays for human CD133 (RealTime Designer Assay code 05583055001; Roche Molecular Biochemicals), according to the manufacturer's instructions. All reactions were performed in triplicate. The thermal cycling conditions included a step of $20 \mathrm{sec}$ at $95^{\circ} \mathrm{C}$ followed by 40 cycles of $95^{\circ} \mathrm{C}$ for $1 \mathrm{sec}$ and $60^{\circ} \mathrm{C}$ for $20 \mathrm{sec}$. The comparative $\mathrm{C}_{\mathrm{t}}$ method was employed to determine the human CD133 gene variation, using as reference gene TaqMan Endogenous Controls Human ACTB ( $\beta$-actin) (Real-Time Designer Assay code 05532957001; Roche Molecular Biochemicals). We identified a calibrator cell line that represents the unitary amount of the target of interest. Consequently, the samples express n-fold mRNA relative to the calibrator. Final amounts of target were determined as follows: Target amount $=2-C_{t}$, where $C_{t}=\left[C_{t}\right.$ $\left.(\mathrm{CD} 133)-\mathrm{C}_{\mathrm{t}}(\mathrm{ACTB})\right]_{\text {sample }}-\left[\mathrm{C}_{\mathrm{t}}(\mathrm{CD} 133)-\mathrm{C}_{\mathrm{t}}(\mathrm{ACTB})\right]_{\text {calibrator }}$.

Statistical analysis. The primary outcome variables were overall survival (OS) and disease-free survival (DFS). Survival curves were estimated using the Kaplan-Meier method, and differences among groups were analyzed using the log-rank test. OS was calculated as the time from the date of surgical resection to death of any cause or until the date of the last follow-up at which point data were censored. DFS was defined as the time between surgical resection until signs of disease progression (PD), death (DCD) from any cause and last radiological assessment. Data on survivors were censored at the last follow-up. We used non-parametric tests to compare independent groups of numerical data (Mann-Whitney test) and categorical data ( $\chi^{2}$-test and Fisher's exact test). The correlations among numerical variables were assessed by Spearman's rank correlation analysis. Multivariate analyses, with backward variable selection, were conducted using the Cox's proportional-hazard regression model. Variables at the 0.10 level in univariate analysis were included in the multivariate model. The level of significance was defined as $\mathrm{P}<0.05$. All the statistical analyses were carried out using the Statistical Package for the Social Science 8.0 software (SPSS Inc., Chicago, IL, USA).

\section{Results}

Cytokeratin expression in CTCs in tumor-draining venous blood and TMA analyses. Cells positive for cytokeratins were 


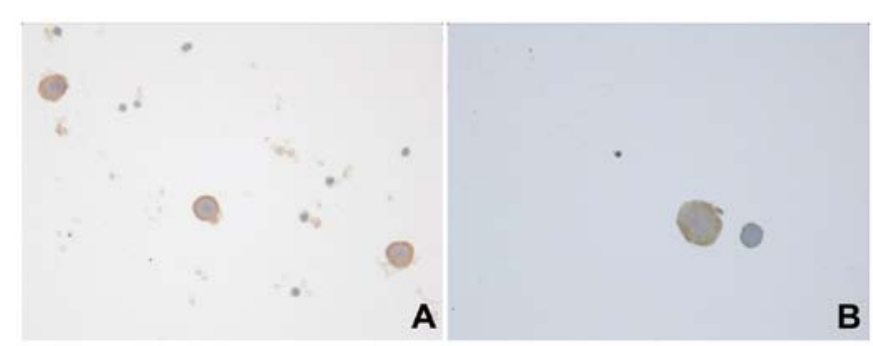

Figure 1. Immunohistochemical analyses for cytokeratins. (A) CTCs positive for pan-cytokeratins (original magnification, $x 400$ ). (B) Detailed image of CTCs positive for pan-cytokeratins (original magnification, x630).
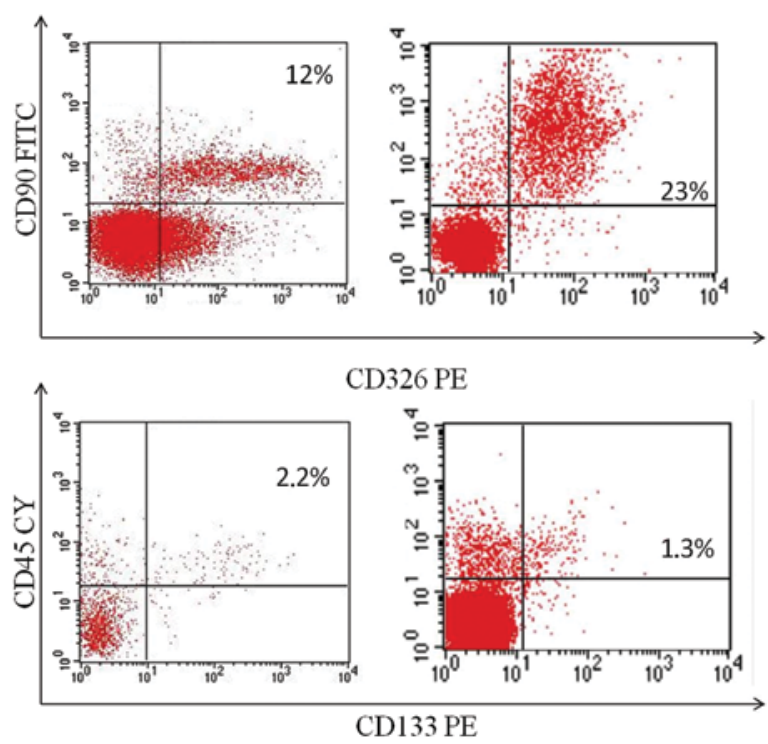

Figure 2. Cytometric analyses of fresh biopsy samples demonstrating expression of CD133 marker and co-expression of CD90/CD326 markers in 2 samples tested.

detectable in $11(23.9 \%)$ cases from tumor-draining pulmonary venous blood and were distributed as showed in Table I. The number of cells positive for cytokeratins ranged from 2 to 10 (Fig. 1).

Flow cytometric analysis and prognosis. Fresh surgical biopsies of NSCLC obtained from 45 patients undergoing tumor resection were mechanically disaggregated, and the resulting single-cell suspensions were analyzed by flow cytometry to identify both the cell population expressing the stemness marker CD133, and the cell population expressing EMT markers CD90 (mesenchymal marker) and CD326 (epithelial marker). The co-expression of CD90 and CD326 identifies the EMT subpopulation. The co-expression of CD90/CD326 markers was detectable in 42 of the $45(93.3 \%)$ samples with a mean percentage of $10.41 \%$ (range, $0-55.2 \%$ ) (Fig. 2). The expression of the CD133 marker was detectable in 40 of the $45(88.8 \%)$ samples with a mean percentage of $1.6 \%$ (range, $0-9.22 \%)$.

TMA evaluation and RT-PCR. Prognostic TMA analyses showed a heterogeneous expression of c-kit as detected by

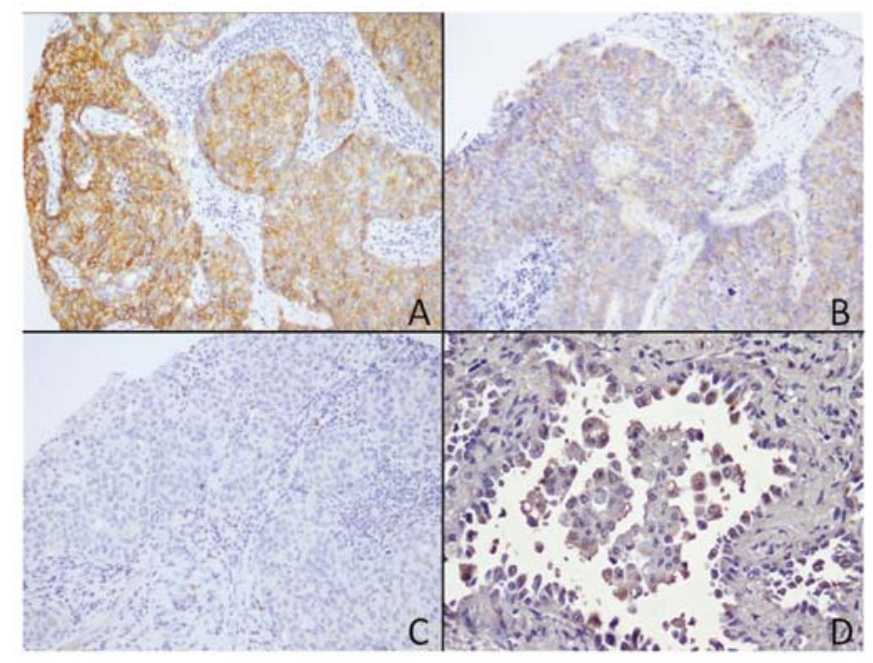

Figure 3. TMA analyses showing (A) high expression of c-kit (original magnification, x200), (B and C) low expression of c-kit (original magnification, $\mathrm{x} 200$ ) and (D) focal expression of CD133 (original magnification, $\mathrm{x} 400$ ).

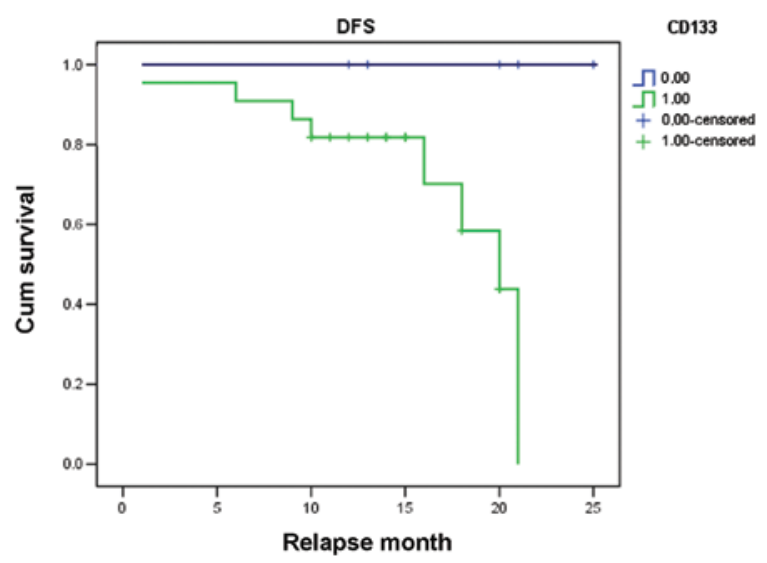

Figure 4. Survival curve showing that patients with tumors positive for CD133 had poorer prognoses than patients with tumors negative for CD133.

immunohistochemistry. In particular, 12 of the 45 cases were positive. CD133 was observed as positive focal membrane expression in neoplastic cells (Fig. 3), and CD133 mRNA was overexpression as detected by qRT-PCR, in all cases documented as positive in cytofluorimetry.

Statistical analysis. Using cytometry, the CD133 antigen showed a high statistically significant correlation with deceased patients when compared with CD90/CD326 co-expression (32.5 vs. 9.5\%, $\mathrm{P}=0.01$ ). Additionally, $\mathrm{CD} 133$ expression exhibited a strong correlation, although not statistically significant, with patients with progression disease when compared to CD90/CD326 co-expression (15 vs. 7.1\%, P=0.21) (Table II). A statistically significantly inverse correlation $(\mathrm{P}=0.04)$ was also noted between CD133 expression and that of c-kit. Moreover, no statistically significant relationship existed between the presence of CTCs and CD133 expression.

CD133 expression was significantly related to shorter DFS $(\mathrm{P}=0.001)$ (Fig. 4). A non-significant relationship was noted between OS and all of the analyzed markers. 
Table II. Correlation among CSCs positive for CD133, EMT markers and patient prognosis.

\begin{tabular}{|c|c|c|c|c|}
\hline & $\operatorname{DCD}(\%)$ & $\mathrm{P}$-value & $\mathrm{PD}(\%)$ & P-value \\
\hline Patients expressing CD133 & 32.5 & 0.01 & 15 & 0.21 \\
\hline Patients co-expressing CD90/CD326 & 9.5 & & 7.1 & \\
\hline
\end{tabular}

DCD, deceased; PD, progressive disease.

\section{Discussion}

Lung cancer is the leading cause of cancer-related deaths worldwide. Generally, the overall survival of lung cancer patients is low, due to late presentation, frequent tumor relapse and lack of effective systemic therapy. Recently, the CSC theory proposes that cancer maintenance is guaranteed by tumor cells that possess stem or progenitor cell features. Thus, CSCs are able to initiate tumor formation and differentiate along multi-potent pathways. In addition, they are relatively resistant to conventional chemotherapy (17). Various stem cell markers of normal tissues have been proposed to detect CSCs. In particular, the CD133 antigen appears to be the most frequently demonstrated marker in liver, brain, colon and lung cancers $(5,7,18,19)$. Thus, recent studies using NSCLC cell lines and fresh lung tumor tissues suggest CD133 as a lung CSC marker (7,8,20,21-23). A biochemical study showed that CD133 plays a functional role in cell cycle regulation and proliferation but not in tumor initiation (24). Yet, numerous other markers have been identified in lung cancer CSCs, with conflicting results. Thus, the marker profile of lung CSCs remains to be explored. Studies performed on lung cancer cell lines by flow cytometry demonstrated no significant CD133 expression by either semi-quantitative RT-PCR, IHC, immunoblotting or flow cytometry analysis. For example, only $0.7 \%$ of cells with CD133 expression was noted in H1299 cells, while no expression was found in our study $(20,25)$. Our data confirmed the relative low percentage of cells positive for CD133 as identified by flow cytofluorimetry in fresh lung cancer tissues.

The relationship between CD133 expression and patient prognosis has been documented in many tumor types. Thus, CD133 expression is related to poor prognosis in cancer of the colorectum (26-29), brain (30), liver (31), stomach (32), endometrium (33), ovary (34) and lung (35). The majority of these studies are small in sample size and are further limited by use of the immunohistochemistry method, which results generally in high background noise with the commercial antibody. Several of the studies utilized CD133 mRNA alone or in combination with other markers. In our study, we evaluated CD133 expression using three different methods, with substantial superimposable results. In fact, cases with CD133 expression by flow cytometry were confirmed by immunohistochemical analysis on paraffin-embedded tumor tissue and by real-time PCR. Although a small series was studied, the use of different methods reinforced the finding that CD133 expression is a significant predictor of lung cancer prognosis.

Other markers have been proposed as CSC biomarkers in lung cancer. Among these, c-kit receptors and embryonic markers have been suggested (36).
In particular, c-kit has been considered a stemness biomarker in many tumor histotypes, with conflicting results in term of prognosis in small and non-small cell lung cancer. In our series we observed no relationship between c-kit expression and patient prognosis, and a significantly inverse relation was noted between c-kit-expressing and CD133-expressing cases. The significance of stemness in cancer varies from one tumor to another, and research aimed to determine an accurate profile of CSCs for each tumor type appears to be the next frontier to elucidate the role of this biological feature in the clinical setting for each patient. In fact, in our series, CD133 was a strong predictor and c-kit was not. Moreover, using immunohistochemistry, these two markers did not identify the same neoplastic cells, probably due to the fact that c-kit expression in this context was not related to stemness properties but to other biological events, such as gene mutations that are described in other tumors.

It is well known that DTCs can be found in the circulation of patients with metastatic cancer, and it has been hypothesized that these DTCs may represent cancer stem cells or a cell population with high metastatic potential (37). In recent years, significant effort has been made to develop technologies that achieve specific and sensitive detection and capture of CTCs. In a recent study (16), we identified in the same set of patients CTCs by immunohistochemistry in pulmonary vein blood obtained after the surgical intervention. No association was found between the presence of CTCs and patient prognosis. Herein, we found that no statistical relationship exists between the presence of CTCs and CD133 expression. This is probably due to the fact that all CTCs have stemness properties, responsible for circulation and colonization to distant organs and recirculation, i.e. to propagate tumor progression. In fact, the majority of CTCs will either die in the bloodstream due to mechanical shear forces, immune surveillance, and/or other regulatory mechanisms; a few cells will successfully extravasate and form new colonies at distant sites. Based on this view, only CSCs are able to survive the difficulties of metastatic development. For this reason, clinical reports evaluating CTCs have provided contradictory results with various studies indicating that CTC detection may be associated with poor prognosis $(38,39)$ whereas others have failed to show such an association (40).

In this context, it is important to consider the role of EMT in metastases. In the present study, we evaluated cells undergoing EMT using CD90 and CD326 markers from cells derived from fresh biopsy samples. CD90 is a main mesenchymal marker, while CD326 is an epithelial marker expressed both in normal and pathological epithelial tissues. Their co-expression indicates cells expressing both epithelial and mesenchymal 
markers; hence, cells undergoing EMT. Our results demonstrated that this cell subset existed, but in respect to CD133 expression, it was not correlated with patient prognosis. In conclusion, our data reinforce the role of CD133 expression in primary tumors in terms of prognosis, although a complete profile of CSCs in lung cancer is needed for correct patient stratification. In addition, the lack of association between the presence of CTCs and CD133 suggests that numerous factors are responsible, but only stemness favors tumor growth in distant sites and cancer maintenance.

\section{Acknowledgments}

The authors would like to thank Dr Alessandra Trocino, librarian of the National Cancer Institute, Naples, for providing excellent bibliographic services and assistance. This study was financially supported by the Italian Government. This study was presented at the 48th Annual Meeting of The Society of Thoracic Surgeons, Fort Lauderdale, FL, February, 2012.

\section{References}

1. Parkin DM, Bray F, Ferlay J and Pisani P: Global cancer statistics, 2002. CA Cancer J Clin 55: 74-108, 2005.

2. Jordan CT, Guzman ML and Noble M: Cancer stem cells. N Engl J Med 355: 1253-1261, 2006.

3. Visvader JE and Lindeman GJ: Cancer stem cells in solid tumours: accumulating evidence and unresolved questions. Nat Rev Cancer 8: 755-768, 2008.

4. Locke M, Heywood M, Fawell S and Mackenzie IC: Retention of intrinsic stem cell hierarchies in carcinoma-derived cell lines. Cancer Res 65: 8944-8950, 2005.

5. Singh SK, Clarke ID, Terasaki M, et al: Identification of a cancer stem cell in human brain tumors. Cancer Res 63: 5821-5828, 2003.

6. Ponti D, Costa A, Zaffaroni N, Pratesi G, et al: Isolation and in vitro propagation of tumorigenic breast cancer cells with stem/progenitor cell properties. Cancer Res 65: 5506-5511, 2005

7. Eramo A, Lotti F, Sette G, et al: Identification and expansion of the tumorigenic lung cancer stem cell population. Cell Death Differ 15: 504-514, 2008.

8. Tirino V, Camerlingo R, Franco R, et al: The role of CD133 in the identification and characterisation of tumour-initiating cells in non-small-cell lung cancer. Eur J Cardiothorac Surg 36: 446-453, 2009.

9. Chambers AF, Groom AC and MacDonald IC: Dissemination and growth of cancer cells in metastatic sites. Nat Rev Cancer 2 : 563-572, 2002.

10. Bonnomet A, Brysse A, Tachsidis A, et al: Epithelial-tomesenchymal transitions and circulating tumor cells. J Mammary Gland Biol Neoplasia 15: 261-273, 2010.

11. Larue L and Bellacosa A: Epithelial-mesenchymal transition in development and cancer: role of phosphatidylinositol 3 kinase/AKT pathways. Oncogene 24: 7443-7454, 2005.

12. Pantel $K$ and Brakenhoff RH: Dissecting the metastatic cascade. Nat Rev Cancer 4: 448-456, 2004.

13. Klein CA: Parallel progression of primary tumours and metastases. Nat Rev Cancer 9: 302-312, 2009.

14. Aguirre-Ghiso JA: Models, mechanisms and clinical evidence for cancer dormancy. Nat Rev Cancer 7: 834-846, 2007.

15. Pantel K, Alix-Panabières $C$ and Riethdorf $S$ : Cancer micrometastases. Nat Rev Clin Oncol 6: 339-351, 2009.

16. Franco R, Pirozzi G, Scala S, Cantile M, Scognamiglio G, Camerlingo R, Botti G and Rocco G: CXCL12-binding receptors expression in non-small cell lung cancer relates to tumoral microvascular density and CXCR4 positive circulating tumoral cells in lung draining venous blood. Eur J Cardiothorac Surg 41: 368-375, 2012.

17. Dick JE: Future prospects for animal models created by transplanting human haematopoietic cells into immune-deficient mice. Res Immunol 145: 380-384, 1994.
18. Ma S, Chan KW, Hu L, et al: Identification and characterization of tumorigenic liver cancer stem/progenitor cells. Gastroenterology 132: 2542-2556, 2007.

19. Ricci-Vitiani L, Lombardi DG, Pilozzi E, et al: Identification and expansion of human colon-cancer-initiating cells. Nature 445: 111-115, 2007.

20. Chen YC, Hsu HS, Chen YW, et al: Oct-4 expression maintained cancer stem-like properties in lung cancer-derived CD133positive cells. PLoS One3: e2637, 2008.

21. Bertolini G, Roz L, Perego P, Tortoreto M, Fontanella E, et al: Highly tumorigenic lung cancer $\mathrm{CD}_{133^{+}}$cells display stem-like features and are spared by cisplatin treatment. Proc Natl Acad Sci USA 106: 16281-16286, 2009.

22. Levina V, Marrangoni AM, DeMarco R, Gorelik E and Lokshin AE: Drug-selected human lung cancer stem cells: cytokine network, tumorigenic and metastatic properties. PLoS One 3: e3077, 2008.

23. Basak SK, Veena MS, Oh S, Huang G, Srivatsan E, et al: The malignant pleural effusion as a model to investigate intratumoral heterogeneity in lung cancer. PLoS One 4: e5884, 2009.

24. Wu Y and Wu PY: CD133 as a marker for cancer stem cells: progresses and concerns. Stem Cells Dev 18: 1127-1134, 2009.

25. Leung EL, Fiscus RR, Tung JW, et al: Non-small cell lung cancer cells expressing CD44 are enriched for stem cell-like properties. PLoS One 5: e14062, 2010.

26. Li CY, Li BX, Liang Y, et al: Higher percentage of $\mathrm{CD} 133^{+}$cells is associated with poor prognosis in colon carcinoma patients with stage IIIB. J Transl Med 7: 56, 2009.

27. Xi HQ and Zhao P: Clinicopathological significance and prognostic value of EphA3 and CD133 expression in colorectal carcinoma. J Clin Pathol 64: 498-503, 2011.

28. Artells R, Moreno I, Diaz T, et al: Tumour CD133 mRNA expression and clinical outcome in surgically resected colorectal cancer patients. Eur J Cancer 46: 642-649, 2010.

29. Horst D, Kriegl L, Engel J, et al: CD133 expression is an independent prognostic marker for low survival in colorectal cancer. Br J Cancer 99: 1285-1289, 2008.

30. Metellus P, Nanni-Metellus I, Delfino C, et al: Prognostic impact of CD133 mRNA expression in 48 glioblastoma patients treated with concomitant radiochemotherapy: a prospective patient cohort at a single institution. Ann Surg Oncol 18: 2937-2945, 2011.

31. Sasaki A, Kamiyama T, Yokoo H, et al: Cytoplasmic expression of CD133 is an important risk factor for overall survival in hepatocellular carcinoma. Oncol Rep 24: 537-546, 2010.

32. Ishigami S, Ueno S, Arigami T, et al: Prognostic impact of CD133 expression in gastric carcinoma. Anticancer Res 30: 2453-2457, 2010.

33. Nakamura M, Kyo S, Zhang B, et al: Prognostic impact of CD133 expression as a tumor-initiating cell marker in endometrial cancer. Hum Pathol 41: 1516-1529, 2010.

34. Silva IA, Bai S, McLean K, et al: Aldehyde dehydrogenase in combination with CD133 defines angiogenic ovarian cancer stem cells that portend poor patient survival. Cancer Res 71: 3991-4001, 2011.

35. Woo T, Okudela K, Mitsui H, et al: Prognostic value of CD133 expression in stage I lung adenocarcinomas. Int J Clin Exp Pathol 4: 32-42, 2010.

36. Levina V, Marrangoni A, Wang T, et al: Elimination of human lung cancer stem cells through targeting of the stem cell factor-c-kit autocrine signaling loop. Cancer Res 70: 338-346, 2010.

37. Maheswaran S and Haber DA: Circulating tumor cells: a window into cancer biology and metastasis. Curr Opin Genet Dev 20: 96-99, 2010.

38. Gudemann CJ, Weitz J, Kienle P, et al: Detection of hematogenous micrometastasis in patients with transitional cell carcinoma. $\mathrm{J}$ Urol 164: 532-536, 2000.

39. Naito T, Tanaka F, Ono A, et al: Prognostic impact of circulating tumor cells in patients with small cell lung cancer. J Thorac Oncol 7: 512-519, 2012.

40. Guzzo TJ, McNeil BK, Bivalacqua TJ, Elliott DJ, Sokoll LJ and Schoenberg MP: The presence of circulating tumor cells does not predict extravesical disease in bladder cancer patients prior to radical cystectomy. Urol Oncol 30: 44-48, 2012. 